\title{
Sensing Throughput Optimization in Cognitive Fading Multiple Access Channels With Energy Harvesting Secondary Transmitters
}

\author{
Sinchan Biswas, Amirpasha Shirazinia and Subhrakanti Dey
}

\begin{abstract}
The paper investigates the problem of maximizing the expected achievable sum rate in a fading multiple access cognitive radio network when secondary user (SU) transmitters have energy harvesting capability, and perform cooperative spectrum sensing. We formulate the problem as maximization of throughput of the cognitive multiple access network over a finite time horizon subject to a time averaged interference constraint at the primary user (PU) and almost sure energy causality constraints at the SUs. The problem is a mixed integer nonlinear program with respect to two decision variables, namely, spectrum access decision and spectrum sensing decision, and the continuous variables sensing time and transmission power. In general, this problem is known to be NP hard. For optimization over these two decision variables, we use an exhaustive search policy when the length of the time horizon is small, and a heuristic policy for longer horizons. For given values of the decision variables, the problem simplifies into a joint optimization on SU transmission power and sensing time, which is non-convex in nature. We present an analytic solution for the resulting optimization problem using an alternating convex optimization problem for non-causal channel state information and harvested energy information patterns at the SU base station (SBS) or fusion center (FC) and infinite battery capacity at the SU transmitters. We formulate the problem with causal information and finite battery capacity as a stochastic control problem and solve it using the technique of dynamic programming. Numerical results are presented to illustrate the performance of the various algorithms.
\end{abstract}

\section{INTRODUCTION}

Spectrum scarcity is a significant issue in modern wireless networks. This is due to the legacy of static allocation policy of the radio spectrum, which prohibits unlicensed users to exploit licensed spectrum even when it is idle. As a solution, the paradigm of cognitive radio (CR) [1] has been proposed. In the interweave paradigm of CR, unlicensed SUs can access the PU licensed spectrum when the PU is idle. The SUs have to vacate the licensed spectrum as soon as the PU becomes active. To achieve this, the SUs sense the spectrum to check whether the PU is active or not. The decision about spectrum sensing is then taken in a cooperative manner by sending all local spectrum sensing decision to an FC which makes an overall decision regarding spectrum access.

The issue of energy efficiency is also a very important aspect of wireless transmission. While traditionally mobile devices have relied on rechargeable batteries, in many situations, periodic battery replacement of the wireless nodes is not a feasible option in practice, such as in sensor networks. Thus energy harvesting from natural sources like wind or solar power is a viable and cost-effective solution for replenishing energy. Recently, there has been significant research in the domain of energy harvesting in wireless environment [2]. With traditional battery limited transmission, ergodic throughput with different wireless channel models has been investigated in [3], while sensing-throughput tradeoff for a single CR user

\footnotetext{
The authors are with the Division of Signals \& Systems, Uppsala University, Sweden, e-mail: subhrakanti.dey@signal.uu.se
}

has also been studied rigorously in [4]. Capacity analysis of wireless system with energy harvesting capability has been studied in [5] as well. Energy harvesting in CR network has also been investigated in [6], [7], and recently in [8], where the authors have investigated achievable throughput optimization in energy harvesting CR networks with respect to sensing time and sensing threshold.

In this paper, we investigate a sensing-throughput optimization problem for a block fading multiple access CR network with a single PU, where all CR nodes (or SUs) are equipped with energy harvesting capability, and perform cooperative spectrum sensing. In this work, we investigate the tradeoff between sensing time and throughput of the CR multiple access channel with respect to transmission power and sensing time, keeping the sensing threshold fixed. Since spectrum sensing also consumes energy, due to the unreliable nature of harvested energy patterns, the CR nodes must make an initial decision as to whether they would perform spectrum sensing or not. All participating CR nodes' sensing decisions are combined at the SBS (also performing as the FC) to arrive at a final spectrum access decision which is broadcast to all $\mathrm{CR}$ nodes. If the $\mathrm{PU}$ is deemed to be absent, the participating CR nodes use the remaining time in each fading block for information transmission with a suitable transmission power. The problem we investigate is a mixed integer nonlinear programming (MINLP) problem with respect to the individual spectrum sensing (whether or not to participate) and the overall spectrum access decisions, sensing time and transmission power of each user. We consider both non-causal and causal channel state information (CSI) and harvested energy information (or information on the battery level at each user) at the FC/SBS, for optimizing the CR multiple access channel throughput over a finite time horizon, where each user's energy consumption in each fading block cannot exceed their battery level at the beginning of the block (energy causality constraint), a peak transmission power constraint and an average interference constraint at the PU receiver.

Our contributions are listed as follows: (i) We investigate the problem of throughput maximization in a multiple access cognitive network environment with energy harvesting and both infinite/finite battery settings. We first derive an analytic closed form solution for the general horizon problem for the case of non-causal CSI using an alternating convex optimization approach converging to a locally optimum solution, (ii) For short horizon lengths, we employ an exhaustive search to find the optimal values of the Boolean variables, namely the spectrum sensing and access decisions, whereas for longer horizons, motivated by the NP hard nature of the problem, we propose a heuristic algorithm to determine these variables in a suboptimal manner, (iii) The problem involving finite battery and causal CSI and harvested energy information is solved using the dynamic programming (DP) algorithm with discretized levels of power and sensing times, (iv) and finally, illustrative numerical results are presented to demonstrate the 
comparative performance of the various algorithms proposed in this work. Due to limited space, all proofs are omitted and can be found in a longer version of this work (available online) [9].

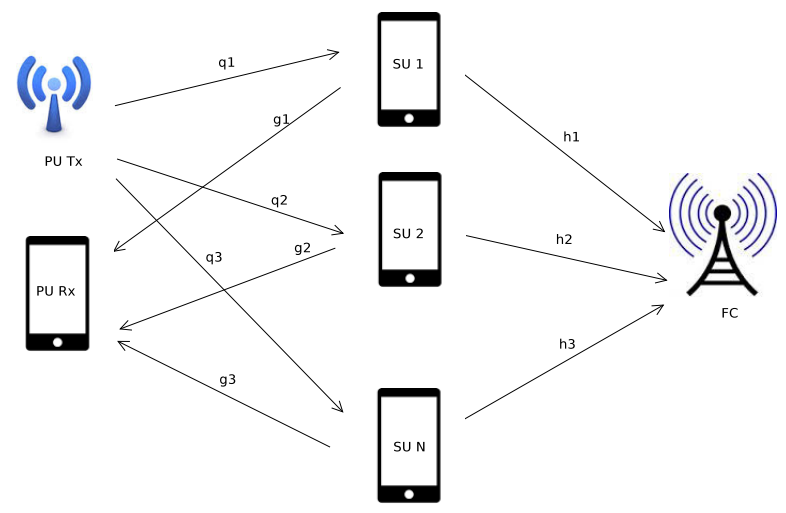

Figure 1: System model for Cognitive MAC.

\section{System MOdel AND PROBlem FORMUlation}

We study the system model as depicted in Fig.1. The system under consideration has $N$ number of SUs communicating to the SBS/FC. In the adopted cooperative spectrum sensing model each SU decides whether to participate or not in sensing the PU spectrum (due to the random nature of harvested energy), and sends its individual spectrum sensing decision (if participating) to the $\mathrm{FC}$, which makes the overall decision and broadcasts it to all the SUs.

We assume that time is slotted, where each slot represents a block where the all fading channels remain invariant and change from one block to another in a statistically independent manner. All relevant parameters, random variables and optimization variables used in this paper are described in Table I. Each time slot of $T$ time units is utilized by the SUs for the spectrum sensing and data transmission. In the $k^{\text {th }}$ time slot SUs spend $\tau_{k}$ time units for spectrum sensing and remainder of the slot $\left(T-\tau_{k}\right)$ time units for data transmission, provided the spectrum sensing result indicates PU spectrum vacancy. In the $k^{\text {th }}$ time slot, $i^{\text {th }}$ SU first makes a decision to perform spectrum sensing or to be idle on the basis of the individual decision to sense $a_{i, k}, 1 \leq i \leq N, 1 \leq k \leq M$, where $M$ is the length of time horizon over which the system performance is optimized, and $a_{i, k} \in\{0,1\}, 1$ (or 0 ) representing the decision to perform (or not to perform) spectrum sensing. The decision to sense $a_{i, k}$ is obtained by the individual SUs by the following rule [10] $a_{i, k}=1$, if $B_{i, k} \geq p_{s} \tau_{k}$ and 0 otherwise, where $p_{s}$ is the sensing power and $B_{i, k}$ is the battery state in the $i^{t h} \mathrm{SU}$ at the beginning of the $k^{t h}$ slot. With a battery of finite capacity $B_{\max }, B_{i, k}$ can be expressed as:

$$
B_{i, k+1}=\min \left\{B_{\max }, B_{i, k}-E_{i, k}+H_{i, k}\right\}
$$

where $H_{i, k}$ is the energy harvested by the $i^{\text {th }} \mathrm{SU}$ in the $k^{\text {th }}$ time slot. In (1), $E_{i, k}$ is the amount of energy used by $i^{\text {th }} \mathrm{SU}$ at $k^{t h}$ time slot, which can be expressed as $E_{i, k}=a_{i, k}\left(p_{s} \tau_{k}+\right.$ $\left.p_{i, k}\left(T-\tau_{k}\right)\left(1-\theta_{k}\right)\right)$, where $\theta_{k}$ is the overall spectrum access decision indicating PU is present if $\theta_{k}=1$ (details in the next subsection). One can also express $B_{i, k}$ as

$$
B_{i, k}=\min \left\{B_{\max }, B_{i}-\sum_{r=1}^{k} E_{i, r}+\sum_{r=1}^{k-1} H_{i, r}\right\}
$$

where $B_{i}$ is the initial battery level of $i^{t h}$ SU. Note that in the infinite battery scenario $B_{i, k}$ from (2) simplifies to $B_{i, k}=$ $B_{i}-\sum_{r=1}^{k} E_{i, r}+\sum_{r=1}^{k-1} H_{i, r}$.

\section{A. Spectrum Sensing Model}

If an SU decides to sense, it collects the samples of the received signal from PU by dividing sensing time interval $\tau_{k}$ to a number of mini-slots, where the length of the mini-slots is a constant and pre-decided. The PU spectrum availability is decided by the following received signal model under hypothesis $\mathcal{H}_{0}$ (PU absent) and $\mathcal{H}_{1}$ (PU present).

$$
\begin{aligned}
& \mathcal{H}_{0}: y_{i, k, m}=n_{i, k, m} \\
& \mathcal{H}_{1}: y_{i, k, m}=q_{i} x_{k}+n_{i, k, m}
\end{aligned}
$$

where $x_{k}$ denotes the PU's transmitted signal for the $k^{t h}$ time slot, where it is assumed to be real valued and distributed as $x_{k} \sim \mathcal{N}\left(0, \sigma_{x}^{2}\right) . q_{i}$ is the CSI between PU transmitter and $i^{t h}$ SU sensing device, which is assumed to be known at the SU throughout the spectrum sensing process. $y_{i, k, m}$ and $n_{i, k, m}$ are the real valued received signal and noise components respectively for the $i^{t h} \mathrm{SU}$, in $k^{t h}$ time slot and $m^{t h}$ minislot. The noise is distributed as $n_{i, k, m} \sim \mathcal{N}\left(0, \sigma_{n}^{2}\right)$. We adopt the energy detection policy of [11] for each SU. The local spectrum sensing decision at the $i^{\text {th }} \mathrm{SU}$ in $k^{t h}$ horizon is defined as $\theta_{i, k}=\mathcal{I}\left\{\frac{1}{S} \sum_{m=1}^{S} y_{i, k, m}^{2} \geq \gamma\right\}$, where $\mathcal{I}$ is the indicator function, $S$ is the number of mini-slots in a particular time slot and $\gamma$ is the detection energy threshold. These local decisions are sent to the FC by error-free control channels and combined using the OR logic fusion. In this scenario, probability of false alarm is $\operatorname{P}_{f a}=\operatorname{Pr}\left\{\theta_{k}=1 \mid \mathcal{H}_{0}\right\}$.

We consider the CSI between the SU transmitters and FC as well as SU transmitters and PU receiver being distributed as exponential random variables with unity mean, without loss of generality. It is also assumed that SUs have the ability of mitigating the interference caused by PU, although the proposed algorithms in this paper can be extended to the case where PU interference can be explicitly considered. We also model the primary user activity as a stationary random

\begin{tabular}{|c|c|}
\hline$\tau_{k}$ & $\begin{array}{l}\text { Time taken to perform the spectrum sensing in the } k^{t h} \\
\text { slot. }\end{array}$ \\
\hline $\begin{array}{l}p_{i, k} \\
\kappa\end{array}$ & $\begin{array}{l}\text { Transmission power for } i^{t h} \mathrm{SU} \text { in the } k^{t h} \text { time slot. } \\
\text { PU activity probability. }\end{array}$ \\
\hline$h_{i, k}$ & $\begin{array}{l}\text { The CSI between } i^{t h} \text { SU Transmitter and FC in the } \\
k^{t h} \text { time slot. }\end{array}$ \\
\hline$g_{i, k}$ & $\begin{array}{l}\text { The CSI between } i^{t h} \text { SU Transmitter and PU Receiver } \\
\text { in the } k^{t h} \text { time slot. }\end{array}$ \\
\hline$q_{i}$ & $\begin{array}{l}\text { The CSI between PU Transmitter and and } i^{t h} \mathrm{SU} \text { in } \\
\text { the } k^{t h} \text { time slot. }\end{array}$ \\
\hline$p_{s}$ & Power allocated to sensing. \\
\hline$P_{\max }$ & The peak power limit on $p_{i, k}$. \\
\hline$B_{i}$ & The initial battery state for $i^{t h}$ user. \\
\hline$H_{i, k}$ & Energy harvested for $i^{t h} \mathrm{SU}$ for the $k^{t h}$ time slot. \\
\hline$a_{i, k}$ & $\begin{array}{l}\text { Spectrum access decision variable for the } i^{\text {th }} \mathrm{SU} \text { in } \\
k^{t h} \text { time slot. }\end{array}$ \\
\hline & Spectrum sensing decision for the $k^{t h}$ horizon. \\
\hline Qavg & The average interference limit to the PU. \\
\hline
\end{tabular}
process, with the probability of the PU being present being $\kappa$ within each time slot.

Table I: System Parameters

\section{B. Throughput Maximization}

For a finite horizon of length $M$, the sensing-throughput optimization problem for a CR multiple access channel with an average interference constraint at the PU receiver, peak SU transmit power constraint, and energy causality constraints can be formulated as (with $1 \leq i \leq N, 1 \leq k \leq M$ )

$$
\max _{a_{i, k}, \theta_{k}, p_{i, k}, \tau_{k}} \mathrm{E}\left\{\sum_{k=1}^{M} \frac{T-\tau_{k}}{M T} \log _{2}\left(1+\sum_{i=1}^{N} p_{i, k} h_{i, k} a_{i, k}\left(1-\theta_{k}\right)\right)\right\}
$$

s.t. $\frac{1}{M} \sum_{k=1}^{M} \mathrm{E}\left\{\frac{T-\tau_{k}}{T} \sum_{i=1}^{N} p_{i, k} g_{i, k} a_{i, k}\left(1-\theta_{k}\right)\right\} \leq Q_{a v g}$

$0 \leq p_{i, k} \leq P_{\max } ; 0 \leq \tau_{k} \leq T ; E_{i, k} \leq B_{i, k}$ a.s. 
Remark The average interference term in the constraint (5) is normalized by the primary activity factor $\kappa$, where $Q_{\text {avg }}=Q / \kappa, Q$ being the actual interference limit, since no interference is caused when the primary is not active. Note also that although the optimization variables indicate $a_{i, k}, \theta_{k}, \tau_{k}$ separately, they are interdependent due to the dependence of $a_{i, k}, \theta_{i, k}$, and $\tau_{k}$.

The above mentioned optimization problem is a MINLP problem with respect to the allocated transmission power $p_{i, k}$, the sensing time $\tau_{k}$, decision to sense $a_{i, k}$ and spectrum sensing result $\theta_{k}$. In principle, one can employ computationally intensive global optimization methods (e.g. see [12]) to solve such NP hard problems. In this paper, for short horizon lengths, we employ an exhaustive search policy for determining the integer variables $a_{i, k}, \theta_{k}$. For longer horizons, we will employ a heuristic policy to be described in the next subsection. For the exhaustive search policy, the optimization is performed at the SBS, and all relevant decision variables are exchanged between the SUs and SBS via control channels.

For a fixed choice of $a_{i, k}$ and $\theta_{k}$, the problem becomes an optimization over $p_{i, k}$ and $\tau_{k}$, which is a non-convex jointly in $p_{i, k}$ and $\tau_{k}$. However, the problem becomes convex if we fix any one of the variables $\tau_{k}$ or $p_{i, k}, \forall i$. We take the approach of alternating convex optimization (see e.g. [13]), where one alternates between optimizing over $\tau_{k}$ with fixed $p_{i, k}$ and vice versa until one reaches a local minimum.

\section{Heuristic Policy for $a_{i, k}$ and $\theta_{k}$}

The exponential complexity of an exhaustive search algorithm for optimizing over $a_{i, k}$ and $\theta_{k}$ can quickly explode for large values of $M$ and $N$. For this scenario, we propose a heuristic suboptimal policy as described below. This heuristic policy is based on the idea that limiting the maximum value of $P_{f a}$ on individual SUs would in turn impose a lower bound $\tau_{l}$ on sensing time for each horizon. If $P_{f a}$ is bounded by the constraint $P_{f a} \leq \alpha$, for some $\alpha>0$, then we can have a corresponding lower bound for $\tau_{k}$ as $\tau_{l} \leq \tau_{k}$, which can be obtained by [4] $\tau_{l}=\frac{1}{f_{s}}\left\{\frac{\mathbf{Q}^{-1}(\alpha)}{\frac{\gamma}{\sigma_{n}^{2}}-1}\right\}^{2}$. where $\mathbf{Q}(\cdot)$ is the complementary distribution function of the standard Gaussian random variable. Then $a_{i, k}=1$ if $B_{i, k}>p_{s} \tau_{l}$ and vice versa. $\theta_{i, k}$ is determined on the basis of the particular $\tau_{k}$ resulting from the alternating convex optimization method, and the final decision $\theta_{k}$ is obtained by combining the individual $\theta_{i, k}$ at the $\mathrm{FC}$ using the OR fusion rule.

\section{III.NON-CAUSAL OPTIMIZATION WITH INFINITE BATTERY}

In this section we analyze the problem first in the context of non-causal CSI $\left(g_{i, k}, h_{i, k}\right)$ and harvested energy $\left(H_{i, k}\right)$ scenario with the assumption of infinite battery, for fixed values of the integer variables $a_{i, k}$ and $\theta_{k}$ (obtained either via exhaustive search or the heuristic policy). We incorporate the aforementioned alternating convex optimization scheme. For the general $M$-horizon problem, we obtain a closed form solution for the optimization problem involving $p_{i, k}$, when $\tau_{k}$ is fixed. We also show that the optimization involving $\tau_{k}$ (when $p_{i, k}$ is fixed) is a linear programming problem, which can be solved by any established LP solver.

\section{A. Optimal Power Allocation Policy}

For a fixed $\tau_{k}$, the optimization problem for transmit power is given below:

$$
\max _{p_{i, k}} \sum_{k=1}^{M} \frac{T-\tau_{k}}{M T} \log _{2}\left(1+\sum_{i=1}^{N} p_{i, k} h_{i, k} a_{i, k}\left(1-\theta_{k}\right)\right)
$$

s.t. $\quad \frac{1}{M} \sum_{k=1}^{M} \frac{T-\tau_{k}}{T} \sum_{i=1}^{N} p_{i, k} g_{i, k} a_{i, k}\left(1-\theta_{k}\right) \leq Q_{\text {avg }}$

$$
0 \leq p_{i, k} \leq P_{\max } ; 1 \leq i \leq N, 1 \leq k \leq M
$$

$$
\sum_{r=1}^{k} a_{i, r}\left(p_{s} \tau_{r}+p_{i, r}\left(T-\tau_{r}\right)\left(1-\theta_{r}\right)\right) \leq B_{i}+\sum_{r=1}^{k-1} H_{i, r}
$$

The Lagrangian of the above mentioned convex optimization problem is given by

$$
\begin{aligned}
& \mathcal{L}\left(\left\{p_{i, k}\right\}, \lambda, \delta_{i, k}, \eta_{i, k}, \mu_{i, k}\right)= \\
& \sum_{k=1}^{M} \frac{T-\tau_{k}}{M T} \times \log _{2}\left(1+\sum_{i=1}^{N} p_{i, k} h_{i, k} a_{i, k}\left(1-\theta_{k}\right)\right) \\
& -\lambda\left(\frac{1}{M} \sum_{k=1}^{M} \frac{T-\tau_{k}}{T} \sum_{i=1}^{N} p_{i, k} g_{i, k} a_{i, k}\left(1-\theta_{k}\right)-Q_{a v g}\right) \\
& +\sum_{k=1}^{M} \sum_{i=1}^{N} \eta_{i, k} p_{i, k}-\sum_{k=1}^{M} \sum_{i=1}^{N} \delta_{i, k}\left(p_{i, k}-P_{\text {max }}\right) \\
& -\sum_{k=1}^{M} \sum_{i=1}^{N} \mu_{i, k}\left(\sum_{r=1}^{k} a_{i, r}\left(p_{s} \tau_{r}+p_{i, r}\left(T-\tau_{r}\right)\left(1-\theta_{j}\right)\right)\right. \\
& \left.-B_{i}-\sum_{r=1}^{k-1} H_{i, r}\right)
\end{aligned}
$$

where $\lambda, \eta_{i, k}, \delta_{i, k}$ and $\mu_{i, k}$ are the non-negative dual variables associated with the average interference constraint, transmission power lower bound, upper bound and the energy causality constraint respectively. Now we define $\mathcal{D}$ as the set of $p_{i, k}$ 's which satisfies (8) and (9). The Lagrange dual function is defined as: $g(\lambda)=\max _{\left\{p_{i, k}\right\} \in \mathcal{D}} \mathcal{L}\left(\left\{p_{i, k}\right\}, \lambda, \eta_{i, k}, \delta_{i, k}, \mu_{i, k}\right)$, and the dual problem is defined as $\min _{\lambda \geq 0} g(\lambda)$. The Lagrange dual function $g(\lambda)$ can be obtained by solving the corresponding optimization problem using the associated Karush-KuhnTucker (KKT) conditions, leading to the expression for $p_{i, k}^{\star}$ by following the same approach as in [3], as described below.

Lemma 1: Let $i$ and $j$ be any two arbitrary users, $i, j \in 1,2, \ldots, N$, with $p_{i, k}^{\star}>0$ and $p_{j, k}^{\star}=0$, then one must have $\frac{c_{i, k}}{\lambda d_{i, k}+e_{i, k}} \geq \frac{c_{j, k}}{\lambda d_{j, k}+e_{j, k}}, \quad, \quad \forall k$, where $c_{i, k}, d_{i, k}, e_{i, k}$ are given by the following expressions: $c_{i, k}=$ $\frac{T-\tau_{k}}{M T \log 2} h_{i, k} a_{i, k}\left(1-\theta_{k}\right), d_{i, k}=\frac{T-\tau_{k}}{M T} g_{i, k} a_{i, k}\left(1-\theta_{k}\right)$, and $e_{i, k}=\sum_{r=k}^{M} \mu_{i, r}^{\star} a_{i, r}\left(T-\tau_{r}\right)\left(1-\theta_{r}\right)$.

Now let $\pi$ be a permutation over $\{1,2, \ldots, N\}$ such that $\frac{c_{\pi(i), k}}{\lambda d_{\pi(i), k}+e_{i, k}} \geq \frac{c_{\pi(j), k}}{\lambda d_{\pi(j), k}+e_{j, k}}$ when $i<j, i, j \in\{1,2, \ldots, N\}$. Suppose there are $|\mathcal{I}|$ users that can transmit, where $\mathcal{I} \subseteq$ $\{1,2, \ldots, N\}$ denotes this set of users. It can be verified that $\mathcal{I}=\{\pi(1), \ldots, \pi(|\mathcal{I}|)\}$. The cardinality of the set $\mathcal{I},|\mathcal{I}|$ is the largest value of $x$ such that

$$
\begin{aligned}
& \frac{c_{\pi(x), k}}{\lambda d_{\pi(x), k}+e_{\pi(x), k}}> \\
& 1+\sum_{b=1}^{x-1} P_{\max } h_{\pi(b), k} a_{\pi(b), k}\left(1-\theta_{k}\right)
\end{aligned}
$$

and the optimal transmission power for $\pi(|\mathcal{I}|)^{t h}$ user is given by the following expression:

$$
\begin{aligned}
& p_{\pi(|\mathcal{I}|), k}^{\star}=\min \left(P_{\text {max }},\left(\frac{c_{\pi(I I \mid), k}}{\lambda d_{\pi(|I|), k}+e_{\pi(|\mathcal{I}|), k}}\right.\right. \\
& \left.-1-\sum_{b=1}^{|\mathcal{I}|-1} P_{\max } h_{\pi(b), k} a_{\pi(b), k}\right) \frac{1}{h_{\pi(|\mathcal{I}|), k}}
\end{aligned}
$$

Note that the solution for $p_{\pi(c), k}^{\star}$ is $P_{\max }$ if $c<|\mathcal{I}|$. If $c>|\mathcal{I}|$, then $p_{\pi(c), k}^{\star}=0$. Only for the case when $c=|\mathcal{I}|$, 
then $p_{\pi(c), k}^{\star}$ is given by (12).

As an example if we consider a specific case of $M=2$ and number of SUs $N=3$, then for any horizon if $\tau_{k}$ is fixed, the optimal transmission power would be lie in the open set $\left(0, P_{\max }\right)$ for at most one of the 3 SUs. All the other SUs either transmit with $P_{\max }$ or do not transmit.

\section{B. Optimal Sensing Time Policy}

Fixing the transmission power would result in the optimization problem in $\left\{\tau_{k}\right\}$ :

$$
\begin{aligned}
\max _{\left\{\tau_{k}\right\}} & \sum_{k=1}^{M} \frac{T-\tau_{k}}{M T} \log _{2}\left(1+\sum_{i=1}^{N} p_{i, k} h_{i, k} a_{i, k}\left(1-\theta_{k}\right)\right) \\
\text { s.t. } & \frac{1}{M} \sum_{k=1}^{M} \frac{T-\tau_{k}}{T} \sum_{i=1}^{N} p_{i, k} g_{i, k} a_{i, k}\left(1-\theta_{k}\right) \leq Q_{a v g} \\
& 0 \leq \tau_{k} \leq T ; 1 \leq k \leq M \\
& \sum_{r=1}^{k} a_{i, r}\left(p_{s} \tau_{r}+p_{i, r}\left(T-\tau_{r}\right)\left(1-\theta_{r}\right)\right) \leq B_{i}+\sum_{r=1}^{k-1} H_{i, r}
\end{aligned}
$$

The above optimization problem can be reformulated as a linear program and solved using a typical LP solver.

It can be shown that the objective function for the alternating convex optimization approach is upper bounded and monotonically non-decreasing at each iteration, which indicates that it must converge. Since the original problem is non-convex, this convergence is only guaranteed to reach a local optimum.

\section{CAUSAL OPtIMIZATION WITH FINITE BATTERY}

While the non-causal information pattern is unrealistic, it serves as a benchmark for the more realistic scenario of causal CSI and battery state information with finite battery capacity. The resulting problem can be formulated as a finite horizon stochastic control problem and thus solved by a dynamic programming algorithm. The values of $a_{i, k}$ and $\theta_{k}$ can be found either via an exhaustive search or suboptimally by the previously discussed heuristic policy depending on the complexity concerned with a given scenario.

\section{A. Information Pattern}

During each time slot $k, \mathrm{FC}$ receives the CSI between SU transmitter and PU receiver $\mathbf{g}_{k}=\left\{g_{1, k}, g_{2, k}, \ldots, g_{N, k}\right\}$ causally, either via cooperation from the PU receiver (or base station), or via feedback from a cooperative node located close to the PU receiver. The CSI between SU transmitter and $\mathrm{FC} \mathbf{h}_{k}=\left\{h_{1, k}, h_{2, k}, \ldots, h_{N, k}\right\}$ and the SU battery state information $\mathbf{B}_{k}=\left\{B_{1, k}, B_{2, k}, \ldots, B_{N, k}\right\}$ are assumed to be received by $\mathrm{FC}$ via typical channel estimation techniques and feedback from the SUs to the FC. Information available to FC at $k^{t h}$ horizon is given by the tuple $\mathbf{J}_{k}=\left\{\mathbf{g}_{k}, \mathbf{h}_{k}, \mathbf{B}_{k}, \mathbf{J}_{k-1}\right\}$.

\section{B. Dynamic Programming Algorithm}

The reward function of the stochastic control problem corresponding to a fixed horizon $k$ can be written as

$$
\begin{aligned}
C\left(p_{i, k}, \tau_{k}\right) & =\frac{T-\tau_{k}}{M T} \log _{2}\left(1+\sum_{i=1}^{N} p_{i, k} h_{i, k} a_{i, k}\left(1-\theta_{k}\right)\right) \\
& -\lambda\left(\frac{T-\tau_{k}}{M T} \sum_{i=1}^{N} p_{i, k} g_{i, k} a_{i, k}\left(1-\theta_{k}\right)-Q_{a v g}\right)
\end{aligned}
$$

Here $\lambda$ is the Lagrange parameter corresponding to average interference constraint. We define the feasible set for the optimization variables as: $S=\left\{\left(p_{i, k}, \tau_{k}\right): p_{i, k}, \tau_{k}\right.$ satisfy $\left.(6)\right\}$. With $\lambda$ fixed, the optimal value of transmission power and sensing time can be determined by the following theorem:

Theorem 1. With the initial condition $\mathbf{J}_{1}=\left\{\mathbf{g}_{1}, \mathbf{h}_{1}, \mathbf{B}_{1}\right\}$, the value of the finite horizon finite battery problem with causal information is given by $V_{1}\left(\mathbf{g}_{1}, \mathbf{h}_{1}, \mathbf{B}_{1}\right)$, which can be computed by the backward Bellman dynamic programming equation:

$$
\begin{gathered}
V_{k}(\mathbf{g}, \mathbf{h}, \mathbf{B})=\max _{\left(p_{i, k}, \tau_{k}\right) \in \mathcal{S}}\left[C\left(p_{i, k}, \tau_{k}\right)+\right. \\
\left.\mathrm{E}\left[V_{k+1}\left(\mathbf{g}_{k+1}, \mathbf{h}_{k+1}, \mathbf{B}_{k+1} \mid p_{i, k}, \tau_{k}\right)\right]\right]
\end{gathered}
$$

The solution of the causal optimization problem, which can be computed numerically by searching over discretized values of the optimization variables, is obtained as:

$$
\begin{gathered}
\left\{p_{i, k}^{\star}, \tau_{k}^{\star}\right\}=\operatorname{argmax}_{p_{i, k}, \tau_{k} \in \mathcal{S}}\left[C\left(p_{i, k}, \tau_{k}\right)+\right. \\
\left.\mathbf{E}\left[V_{k+1}\left(\mathbf{g}_{k+1}, \mathbf{h}_{k+1}, \mathbf{B}_{k+1} \mid p_{i, k}, \tau_{k}\right)\right]\right]
\end{gathered}
$$

The optimal value of $\lambda$ from (13) is found by the solving the following equation:

$$
\lambda\left[\mathrm{E}\left\{\sum_{k=1}^{M} \frac{T-\tau_{k}}{T} \sum_{i=1}^{N} p_{i, k} g_{i, k} a_{i, k}\left(1-\theta_{k}\right)\right\}-Q_{a v g}\right]=0
$$

In practice, one can solve for the optimal $\lambda$ using a bisection search based iterative method. In this method, each new iterate of $\lambda$ can be substituted in (14) to find the corresponding optimal $p_{i, k}^{\star}, \tau_{k}^{\star}$, which in turn are substituted in (14) to solve for a new value of $\lambda$ and so on. Convergence of such algorithms follow from monotonicity of the left hand side of (14) in $\lambda$. Details can be found in [9]. Note that this procedure is performed offline purely based on the statistics of the channel gains and harvested energy information. Based on this procedure, the FC creates a lookup table for optimal values of $p_{i, k}$ and $\tau_{k}$ corresponding to discrete quantized values of $\mathbf{g}_{k}, \mathbf{h}_{k}, \mathbf{H}_{k}$. In real time FC receives the channel gains and battery states and check for the closest quantization point in its lookup table. The optimal sensing and transmission power are fetched from the look-up table and sent to the individual SUs, and then used for sensing and information transmission.

\section{Simulation Results}

In this section we present numerical results for the causal and non-causal CSI and battery state scenarios for the optimization problem under consideration. We assume the energy harvesting process is an exponentially distributed random process with unity mean. The PU activity probability is set to $\kappa=0.8$. The sensing channel signal to noise ratio (SNR) is assumed to be $-15 \mathrm{~dB}$. The PU signal variance is taken to be $\sigma_{s}^{2}=1 \mathrm{~mW}$. We use $N=2$ SUs. The length of a time slot is taken as $T=2 \mathrm{~ms}$. The probability of false alarm $P_{f a}$ is taken to be 0.03 . The sampling frequency is assumed to be $1 \mathrm{MHz}$ and the normalized threshold of detection is assumed to be $\frac{\gamma}{\sigma_{n}^{2}}=1.006$. This corresponds to a minimum sensing time limit $\tau_{l}=0.1 \mathrm{~ms}$. For a fair comparison, this constraint on the minimum sensing time has been applied to all of the non-causal, causal and heuristic policy based methods. Transmission power is assumed to be upper bounded by $P_{\max }=1 \mathrm{~mW}$. The initial battery level for each SU is assumed to be $0.4 \mathrm{~mW}$. For dynamic programming $\mathbf{g}_{k}, \mathbf{h}_{k}, \mathbf{H}_{k}$ are quantized into 5 different discrete levels. In Fig.2, we plot the average sensing time for non-causal CSI and battery state scenario denoted by $\tau_{\text {avg }}$ with respect to average harvested energy denoted by $\mu_{H}$, keeping the mean of the channel distribution denoted by $\mu_{g}$ and $\mu_{h}$ constant, for $M=2,3,4$. The averages are taken over 50 Monte-Carlo simulations. From Fig.2, it is evident that average sensing time $\tau_{\text {avg }}$ decreases monotonically by increasing the length of the horizon. This is due to the fact that increasing the length of horizon $M$ in the non-causal CSI and battery state scenario helps to spread out the sensing time over multiple time slots, which means on average transmission time increases and sensing time decreases with increasing $M$. Fig.3 shows 


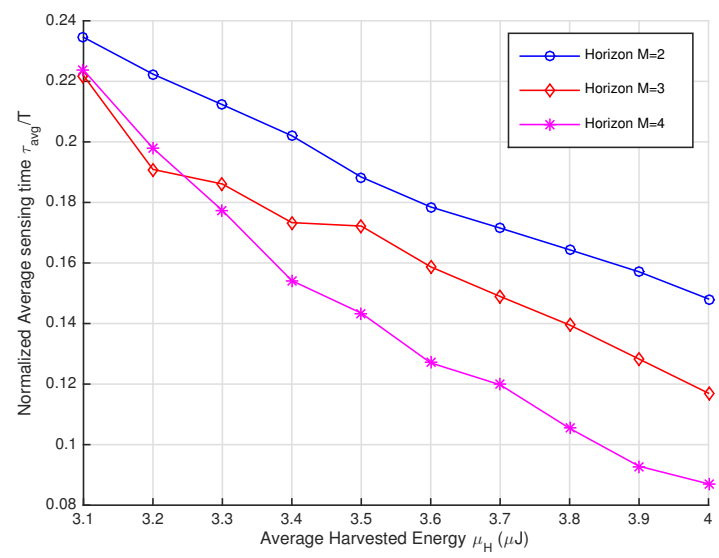

Figure 2: Normalized average sensing time $\tau_{a v g}$ vs Average harvested energy $\mu_{H}$ with ratio of SU Tx-FC Rx and SU Tx-PU Rx average channel gain $\mu_{h} / \mu_{g}$ fixed.

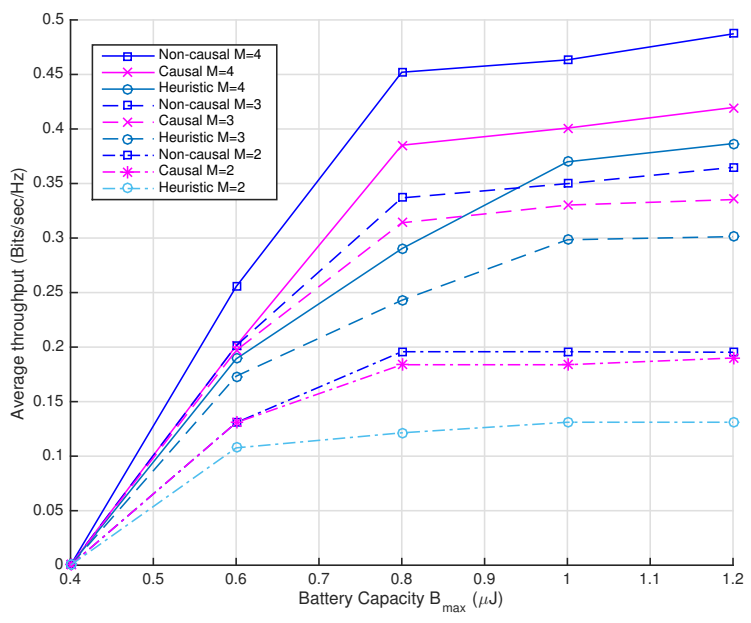

Figure 3: Average throughput vs $B_{\max }$ with causal and non-causal information patterns with $M=2,3,4$.

the optimized average throughput, i.e. average throughput plotted against battery capacity $B_{\max }$ for non-causal CSI and battery state scenario with exhaustive search technique and causal CSI and battery state scenario with exhaustive search and heuristic policy. As expected the average throughput increases with increasing horizon and non-causal CSI and battery state scenario provides an upper bound for the causal counterpart. Also the heuristic policy by choosing $a_{i, k}$ and $\theta_{k}$ performs inferior to both of them, but serves as a less computationally complex alternative to its optimal counterpart. As a numerical comparison, it should be noted that for $M=3$ and $B_{\max }=1 \mu \mathrm{J}$ the average throughput loss with exhaustive search and heuristic policy in causal CSI and battery state scenario compared to non-causal counterpart are 5.7 and 14.7 percent respectively. Fig. 4 shows the heuristic policy-based optimized average throughput as a function of varying battery capacity in the causal CSI and battery state scenario with finite battery. These plots are obtained for three different significantly longer horizon lengths $M=10,15,20$, due to the reduced complexity of the heuristic policy.

\section{VI.CONCLUSION}

This paper investigated a MINLP problem of maximizing the expected achievable sum throughput in a fading multiple access CR network with the energy harvesting constraints where the CR nodes perform cooperative spectrum sensing. An analytical solution is obtained for the system with non-

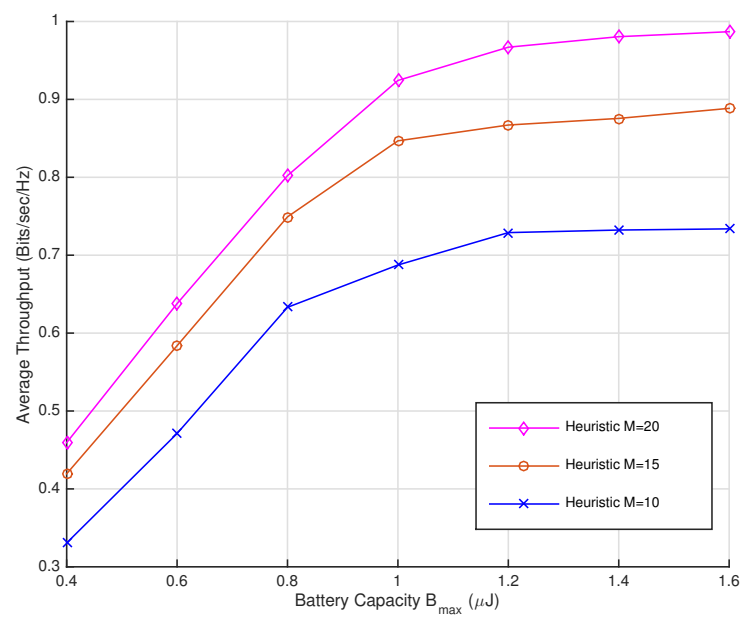

Figure 4: Average throughput vs $B_{\max }$ for the heuristic policy with $M=10,15,20$.

causal CSI and infinite battery capacity. This provides an upper bound on the throughput of a more realistic scenario involving causal CSI and finite battery capacity, which can be formulated as a stochastic control problem and solved using a dynamic programming algorithm. To combat the exponential complexity of handling exhaustive search policies involving the decision variables in the causal CSI scenario, a heuristic policy is proposed. The problem can be extended to incorporate the concept of infinite horizon optimization and energy sharing between the SUs, and also the innovation of better performing heuristic policies.

\section{REFERENCES}

[1] J. Mitola and J. Maguire, G.Q., "Cognitive radio: making software radios more personal," IEEE Personal Communications, vol. 6, no. 4, pp. 13-18, Aug 1999.

[2] K. Tutuncuoglu and A. Yener, "Optimum transmission policies for battery limited energy harvesting nodes," IEEE Transactions on Wireless Communications, vol. 11, no. 3, pp. 1180-1189, March 2012.

[3] R. Zhang, S. Cui, and Y.-C. Liang, "On ergodic sum capacity of fading cognitive multiple-access and broadcast channels," IEEE Transactions on Information Theory, vol. 55, no. 11, pp. 5161-5178, Nov 2009.

[4] Y.-C. Liang, Y. Zeng, E. Peh, and A. T. Hoang, "Sensing-throughput tradeoff for cognitive radio networks," IEEE Transactions on Wireless Communications, vol. 7, no. 4, pp. 1326-1337, April 2008.

[5] O. Ozel and S. Ulukus, "Information-theoretic analysis of an energy harvesting communication system," in IEEE 21st International Symposium on Personal, Indoor and Mobile Radio Communications Workshops (PIMRC Workshops), 2010, Sept 2010, pp. 330-335.

[6] S. Park and D. Hong, "Achievable throughput of energy harvesting cognitive radio networks," IEEE Transactions on Wireless Communications, vol. 13, no. 2, pp. 1010-1022, February 2014.

[7] S. Park, H. Kim, and D. Hong, "Cognitive radio networks with energy harvesting," IEEE Transactions on Wireless Communications, vol. 12, no. 3, pp. 1386-1397, March 2013.

[8] S. Yin, Z. Qu, and S. Li, "Achievable throughput optimization in energy harvesting cognitive radio systems," IEEE Journal on Selected Areas in Communications, vol. 33, no. 3, pp. 407-422, March 2015.

[9] S. Biswas, A. Shirazinia, and S. Dey, "Sensing throughput optimization in cognitive fading multiple access channels with energy harvesting secondary transmitters," feb 2016, available at http://arxiv.org/abs/1602.06722.

[10] W. Chung, S. Park, S. Lim, and D. Hong, "Spectrum sensing optimization for energy-harvesting cognitive radio systems," IEEE Transactions on Wireless Communications, vol. 13, no. 5, pp. 2601-2613, May 2014.

[11] S. Atapattu, C. Tellambura, and H. Jiang, "Energy detection based cooperative spectrum sensing in cognitive radio networks," IEEE Transactions on Wireless Communications, vol. 10, no. 4, pp. 12321241, April 2011.

[12] C. A. Floudas, Nonlinear and Mixed-Integer Optimization. Fundamentals and Applications, 1st ed. Oxford University Press, 1995.

[13] H. Attouch, J. Bolte, P. Redont, and A. Soubeyran, "Alternating minimization and projection methods for nonconvex problems," 0801.1780v2[math.oc], arXiv, 2008. 